\title{
RESEARCH
}

Open Access

\section{Regulation of connective tissue growth factor expression by miR-133b for the treatment of renal interstitial fibrosis in aged mice with unilateral ureteral obstruction}

Dan Cao ${ }^{\dagger}$, Yuan Wang ${ }^{\dagger}$, Yingjie Zhang, Yinping Zhang, Qi Huang, Zhong Yin, Guangyan Cai, Xiangmei Chen and Xuefeng Sun ${ }^{*}$ (1)

\begin{abstract}
Introduction: Renal interstitial fibrosis, an important pathological feature of kidney aging and chronic renal failure, is regulated by mesenchymal stem cells (MSCs). We have previously demonstrated low expression of miR-133b in MSC-derived extracellular vesicles (MSC-EVs) in aged rats. However, miR-133b can mediate the inhibition of epithelial-mesenchymal transition (EMT) of renal tubules induced by transforming growth factor- $\beta 1$ (TGF- $\beta 1$ ). We investigated the effect of miR-133b for the treatment of geriatric renal interstitial fibrosis and evaluated its target genes.

Methods: We performed real-time polymerase chain reaction to detect miR-133b expression induced during EMT of HK2 cells by TGF- $\beta 1$ at different concentrations $(0,6,8$, and $10 \mathrm{ng} / \mathrm{mL})$ and at different time points $(0,24,48$, and $72 \mathrm{~h}$ ). The target genes of miR-133b were validated using the dual-luciferase reporter assay. In vitro experiments were performed to evaluate mRNA and protein expression of miR-133b targets, E-cadherin, a-smooth muscle actin (SMA), fibronectin, and collagen 3A1 (Col3A1), in HK2 cells transfected with miR-133b under TGF- $\beta 1$ stimulation. A 24-month-old unilateral ureteral obstruction (UUO) mouse model was established and injected with transfection reagent and miR-133b into the caudal vein. The target gene of miR-133b and other parameters mentioned above such as mRNA and protein expression levels and renal interstitial fibrosis were detected at 7 and 14 days.

\footnotetext{
* Correspondence: xfssun@126.com

${ }^{\dagger}$ Dan Cao and Yuan Wang contributed equally to this work.

Department of Nephrology, Chinese PLA General Hospital, Chinese PLA Institute of Nephrology, State Key Laboratory of Kidney Diseases, National Clinical Research Center for Kidney Diseases, 28 Fuxing Road, Beijing, China
}

(c) The Author(s). 2021 Open Access This article is licensed under a Creative Commons Attribution 4.0 International License, which permits use, sharing, adaptation, distribution and reproduction in any medium or format, as long as you give appropriate credit to the original author(s) and the source, provide a link to the Creative Commons licence, and indicate if changes were made. The images or other third party material in this article are included in the article's Creative Commons licence, unless indicated otherwise in a credit line to the material. If material is not included in the article's Creative Commons licence and your intended use is not permitted by statutory regulation or exceeds the permitted use, you will need to obtain permission directly from the copyright holder. To view a copy of this licence, visit http://creativecommons.org/licenses/by/4.0/. The Creative Commons Public Domain Dedication waiver (http://creativecommons.org/publicdomain/zero/1.0/) applies to the data made available in this article, unless otherwise stated in a credit line to the data. 
(Continued from previous page)

Results: miR-133b expression gradually decreased with an increase in TGF- $\beta 1$ concentration and treatment time, and the miR-133b mimic downregulated connective tissue growth factor (CTGF) expression. The dual-luciferase reporter assay confirmed CTGF as a direct target of miR-133b. Transfection of the miR-133b mimic inhibited TGF- $\beta 1$ induced EMT of HK2 cells; this effect was reversed by CTGF overexpression. miRNA-133b expression significantly increased (approximately 70-100 times) in mouse kidney tissues after injection of the miRNA-133b overexpression complex, which significantly alleviated renal interstitial fibrosis in mice with UUO.

Conclusion: miR-133b exerted targeted inhibitory effects on CTGF expression, which consequently reduced TGF$\beta 1$-induced EMT of HK2 cells and renal interstitial fibrosis in aged mice with UUO.

Keywords: miR-133b, Target gene, Aged, Renal interstitial fibrosis

\section{Background}

Aging is characterized by significant changes in the structure and function of the kidney, even in the absence of age-related comorbidities. Renal interstitial fibrosis is an important pathological feature of kidney aging [1], and bone marrow mesenchymal stem cells (MSCs) play an important role in the regulation of renal interstitial fibrosis. Transplantation of the bone marrow from young mice into aged mice was shown to significantly reduce renal fibrosis as well as the expression of markers associated with aging in the recipient mice. However, bone marrow cells did not directly replace parenchymal cells, but instead exerted paracrine effects on renal parenchymal cells [2]. Further, the injection of MSCs and MSCderived extracellular vesicles (MSC-EVs) was shown to significantly alleviate renal interstitial fibrosis in a mouse model of unilateral ureteral obstruction (UUO) [3, 4].

Aging can significantly alter the number of stem cells and their regenerative capacity and functions [5]. Our previous research revealed significant differences in the expression profiles of microRNAs (miRNAs) of MSCEVs derived from the bone marrow of young and aged rats. We found that miR-133b-3p was underexpressed in MSC-EVs of aged rats; however, miR-133b could inhibit the epithelial-mesenchymal transition (EMT) of renal tubular cells induced by transforming growth factor (TGF)- $\beta 1$ [6]. The inhibitory effects of MSC-EVs against renal fibrosis decreased with age. miR-133b of MSC-EVs derived from aged rats has been suggested to exhibit important intervening effects on renal fibrosis [7].

miR-133b ameliorates cardiac fibrosis [8-10], reduces TGF- $\beta 1$-mediated EMT of bladder smooth muscle epithelial cells [11], and directly targets the connective tissue growth factor (CTGF) [9, 11]. Whether CTGF is a target involved in miR-133b-mediated inhibition of EMT of renal tubular epithelial cells remains unclear, and studies are warranted to investigate the effect of exogenous expression miR-133b in geriatric renal interstitial fibrosis.

In this study, EMT of the human renal proximal tubular epithelial cell line HK2 was stimulated by TGF- $\beta 1$ in vitro to investigate the role of miR-133b and its target genes in this process. A mouse model of UUO was established in aged C5BL/6J7 mice (aged 24 months), which were then intravenously injected with a miR-133b transfection complex to verify the effect of miR-133b overexpression in geriatric renal fibrosis.

\section{Methods \\ TGF- $\beta 1$ stimulation and miR-133b transfection}

The HK2 cells were cultured in the Dulbecco's modified Eagle medium (DMEM)/F12 (Corning, USA) supplemented with $5 \%$ fetal bovine serum. After reaching $50 \%$ confluency, the cells were synchronized in serum-free DMEM/F12 for $18 \mathrm{~h}$ and then stimulated with TGF- $\beta 1$ at 6,8 , and $10 \mathrm{ng} / \mathrm{mL}$ concentrations for 24,48 , and 72 h. miR-133b mimic and miRNA mimic control (GenePharma, China) were transfected into HK2 cells for $6 \mathrm{~h}$ using the jetPRIME ${ }^{\oplus}$ transfection reagent according to the manufacturer's instructions (Polyplus-transfection, France). Following transfection, the cells were cultured in DMEM/F12 with 5\% serum for $18 \mathrm{~h}$ and then incubated with DMEM/F12 with $5 \%$ serum and $8 \mathrm{ng} / \mathrm{mL}$ of TGF- $\beta 1$ (PeproTech, USA) for $48 \mathrm{~h}$.

\section{RNA extraction and real-time polymerase chain reaction (RT-PCR)}

Total RNA was extracted from HK2 cells and kidney tissues of each group using Trizol and subsequently used to synthesize miR-133b and U6 cDNA using the miScript II RT Kit (QIAGEN, China). Primers specific for target genes were designed with reference to their mRNA-coding regions in GenBank using the Primer 5.0 software. The primer sequences were verified using BLAST. Total RNA was used to synthesize cDNA of target genes using the ReverTra Ace qPCR RT Master Mix kit (TOYOBO, Japan). The expression of genes encoding miR-133b, CTGF, E-cadherin, $\alpha$-smooth muscle actin (SMA), fibronectin, collagen 3A 1 (Col3A1), U6, and glyceraldehyde 3-phosphate dehydrogenase (GAPDH) was detected on the ABI-prism-7500 sequence detection system (Applied Biosystems, USA) using the miScript 
SYBR Green PCR Kit (QIAGEN). The relative expression levels were calculated using $U 6$ or GAPDH as internal control.

\section{Western blot analysis}

After lysis and denaturation of HK2 cells or kidney tissues from each group using the radioimmunoprecipitation assay (RIPA) buffer, proteins $(50 \mu \mathrm{g})$ were separated by $8 \%$ sodium dodecyl sulfate-polyacrylamide gel electrophoresis (SDS-PAGE) and then transferred onto nitrocellulose $(\mathrm{NC})$ membranes. After blocking with $1 \mathrm{x}$ casein for $1 \mathrm{~h}$ to prevent non-specific binding, NC membranes were incubated with the following primary antibodies overnight at $4{ }^{\circ} \mathrm{C}$ : (a) rabbit monoclonal Ecadherin antibody (BD Bioscience, USA) diluted 1:100, (b) rabbit monoclonal anti- $\alpha$-SMA antibody (Abcam, UK) diluted 1:300, (c) mouse monoclonal anti-CTGF antibody (Abcam) diluted 1:200, (d) rabbit polyclonal anti-fibronectin antibody (Proteintech, USA) diluted 1: 500, (e) rabbit polyclonal Col3A1 antibody (Proteintech) diluted 1:500, and (f) mouse monoclonal $\beta$-actin antibody (Beyotime, China) diluted 1:10,000. The membranes were washed with TBST (Tris-buffered saline with Tween-20, $20 \mathrm{mM}$ of Tris, $140 \mathrm{mM}$ of $\mathrm{NaCl}$, and $0.1 \%$ Tween-20) and then probed with 1:1000 diluted secondary antibodies at room temperature $\left(25^{\circ} \mathrm{C}\right)$ for 2 h. Enhanced chemiluminescence (ECL) western blotting kit (APPLYGEN, China) was used to detect the target bands, and $\beta$-actin was used as an internal reference to calculate the relative expression levels of proteins in each experimental group.

\section{Prediction of target genes of miR-133b}

The target genes of miR-133b were predicted using three commonly employed target gene prediction software, namely TargetScan (http://www.targetscan.org/), miRBase (http://www.mirbase.org/), and PicTar (https:// pictar.mdc-berlin.de/).

\section{Dual-luciferase reporter assay}

The seed sequence for the binding of CTGF and miR133b was searched using the bioinformatics software TargetScan. The sequence 5'-AUUUGUUGAGUGUG ACCAAAA-3' containing the $3^{\prime}$-untranslated region (UTR) of CTGF was synthesized and cloned into a luciferase reporter vector GP-miRGLO (GenePharma) and termed as miRGLO-Wt-CTGF. A mutant sequence $5^{\prime}$ AUUUGUUGAGUGUUGGAUUAA-3' of the target was also synthesized and cloned into the plasmid to obtain miRGLO-Mut-CTGF, which was used as the negative control.

293T cells from the logarithmic growth phase were lysed with pancreatin and seeded in 48-well plates for $24 \mathrm{~h}$. After reaching $80 \%$ confluency, the cells were transfected using the cell fusion reagent. The synthesized miR-133b mimic and miRNA mimic control (NC-miR) were co-transfected with miRGLO-Wt-CTGF or miRGLO-Mut-CTGF, respectively, into 293T cells using the jetPRIME transfection reagent according to the manufacturer's instructions. After $48 \mathrm{~h}$, the cells were lysed using a passive lysis buffer (Promega, USA) and the cell lysate was collected. The luciferase activity of the lysate was detected according to the instructions indicated in the dual-luciferase reporter assay system kit (Promega).

\section{Immunofluorescence staining}

HK2 cells were seeded at a density of approximately $10^{5}$ cells/well in 6-well plates with sterile glass coverslips which were disinfected by autoclaving. The plates were placed in an incubator at $37^{\circ} \mathrm{C}$ with $5 \%$ carbon dioxide for $6 \mathrm{~h}$ to allow the cells to adhere to the glass cover slips. After synchronization and transfection as mentioned above, the cells were incubated with DMEM/F12 containing $5 \%$ serum with or without $8 \mathrm{ng} / \mathrm{mL}$ of TGF$\beta 1$ for $48 \mathrm{~h}$. The cells were then fixed with $4 \%$ paraformaldehyde at room temperature for $20 \mathrm{~min}$ and treated with $0.2 \%$ Triton X-100 for 2 min for permeabilization. The cells were then blocked with $5 \%$ bovine serum albumin (BSA) at room temperature for $1 \mathrm{~h}$ and then treated with anti-E-cadherin rabbit monoclonal primary antibody (1:100 dilution), $\alpha$-SMA antibody (1:100 dilution), rabbit polyclonal Col3A1 antibody (1:100 dilution), fibronectin antibody (1:100 dilution), and mouse monoclonal anti-CTGF antibody (1:100 dilution) diluted in 5\% BSA at $4{ }^{\circ} \mathrm{C}$ overnight. After washing with phosphatebuffered saline (PBS), the cells were probed with an antirabbit fluorescein isothiocyanate (FITC)-conjugated fluorescent secondary antibody (Beyotime) (1:400 dilution) at room temperature $\left(25^{\circ} \mathrm{C}\right)$ in the dark for $2 \mathrm{~h}$. The slides were then washed with PBS and treated with 4',6-diamidino-2-phenylindole (DAPI) (ZSGB-BIO, China) fluorescence nuclear staining mounting medium. The expression of $\alpha$-SMA and E-cadherin in HK2 cells from each group was observed under a fluorescence microscope $(\times 100)$ with random fields of vision.

\section{Experimental animals and establishment of the UUO model}

Animal care and experiments were performed according to the guidelines of the Institutional Animal Care and Use Committee of Chinese PLA General Hospital. A total of 28 female C5BL/6J7 mice (SPF grade), aged 24 months, weighing $20 \pm 2 \mathrm{~g}$, were provided by SPF Biotechnology Co., Ltd. (Beijing, China). Mice were randomly divided into sham $(n=8)$, UUO+NC-miR-133b $(n=10)$, and UUO+miR-133b $(n=10)$ groups. 
To establish the UUO model, each mouse was anesthetized by an intraperitoneal injection of pentobarbital $(50 \mathrm{mg} / \mathrm{kg}$ ) and the abdominal cavity was opened under sterile conditions. The left ureter was dissociated, double-ligated, and disconnected at $15 \mathrm{~mm}$ below the renal pelvis with a 4-0 thread. The abdominal cavity was closed by layered suture. In the control group, the abdominal cavity was closed immediately after the ureter was dissociated.

The miR-133b mimic or NC-miR (3 mg/kg) was diluted to $1 \mu \mathrm{g} / \mu \mathrm{L}$ concentration using endotoxin-free purified water. The transfection reagent Entranster-in vivo (Engreen, China) was diluted in a 10\% glucose solution to a final glucose concentration of $5 \%$. The transfection complex was prepared by mixing the two agents and by incubating for $15 \mathrm{~min}$. The UUO+miR-133b and UUO+ NC-miR groups were administered with miR-133b and the NC-miRNA transfection complex $(100 \mu \mathrm{L} /$ animal), respectively, by caudal vein injection at $24 \mathrm{~h}$ before surgery and once every 3 days thereafter. The sham group was administered with the same volume of normal saline by caudal vein injection. The mice were euthanized at 7 and 14 days after the UUO procedure (four mice from the sham group and five mice from the UUO group were euthanized at each time point). The kidney tissues were collected from the obstructed side for western blotting, real-time PCR, and pathological analyses.

\section{Pathological examination of the kidney tissue}

The kidney tissue was fixed in $10 \%$ neutral formaldehyde, dehydrated with ethanol, embedded in paraffin, and cut into $2-\mu \mathrm{m}$ sections. Morphological changes in the kidney tissue were observed by periodic acid-Schiff (PAS) and Masson's trichrome staining. After staining with the Masson's trichrome, 10 fields of vision were selected under a light microscope $(\times 400)$. The area of each field of vision and area of collagen fibers stained in green were measured using the Image-Pro Plus software. The relative area of collagen deposition was calculated as follows: area of collagen fibers stained in green/area of field of vision $\times 100 \%$.

\section{Statistical analysis}

The data were analyzed using the SPSS 17.0 software, and the results were expressed as mean \pm standard error of mean (SEM). The differences among the experimental groups were analyzed using one-way analysis of variance (ANOVA) with a completely random design. Results with $P<0.05$ were considered statistically significant.

\section{Results}

Inhibitory effect of TGF- $\beta 1$ on miR-133b

The real-time PCR results of miR-133b expression analysis showed that the stimulation of HK2 cells with
TGF- $\beta 1$ at concentrations of $0,6,8$, and $10 \mathrm{ng} / \mathrm{mL}$ for $48 \mathrm{~h}$ resulted in a gradual decrease in the expression of miR-133b in a TGF- $\beta 1$ concentration-dependent manner. However, no significant difference was observed between the $8-\mathrm{ng} / \mathrm{mL}$ - and $10-\mathrm{ng} / \mathrm{mL}$-treated groups (Fig. 1A). The expression of miR-133b after stimulation of HK2 cells with $8 \mathrm{ng} / \mathrm{mL}$ of TGF- $\beta 1$ for $0,24,48$, and $72 \mathrm{~h}$ gradually decreased upon increasing stimulation periods; no significant difference was observed between the 48-h and 72-h treatment groups (Fig. 1B).

\section{Effect of miR-133b on the morphology of TGF- $\beta 1$ - stimulated HK2 cells}

Morphological changes in the cells were observed under an inverted microscope. HK2 cells exhibited a round or an oval shape and were arranged in a cobblestone pavement-like pattern. After stimulation with $8 \mathrm{ng} / \mathrm{mL}$ of TGF- $\beta 1$ for $48 \mathrm{~h}$, the cells became slender and fusiform, and their intercellular space significantly widened. While most cells transfected with miR-133b mimic continued to maintain the morphology of epithelial cells, the NC-miR-transfected cells showed the morphology of fibroblasts (Fig. 2).

\section{CTGF is a direct target gene of miR-133b}

We used three different miRNA target gene prediction software (TargetScan, PicTar, and miRbase) and found CTGF to be a potential target of miR-133b. The target site was located between 1027 and $1033 \mathrm{bp}$ of the $3^{\prime}$ UTR of CTGF. The results of DAVID bioinformatics resources suggested that CTGF might be involved in TGF$\beta$-mediated fibrosis and in other signaling pathways.

After transient transfection of a miR-133b mimic into HK2 cells for $48 \mathrm{~h}$, we performed real-time PCR and western blotting and found a significant increase in the miR-133b expression (increased by approximately 21,566 times) (Fig. 3A), which confirmed the successful transfection of miR-133b. While the expression of CTGF mRNA and protein significantly decreased following miR-133b transfection, there was no change in the group transfected with NC-miR (Fig. 3B, C).

miR-133b inhibits EMT of HK2 cells induced by TGF- $\beta 1$ Normal HK2 (untransfected) cells or HK2 cells transfected with miR-133b mimic/NC-miR for $24 \mathrm{~h}$ were stimulated with $8 \mathrm{ng} / \mathrm{mL}$ of TGF- $\beta 1$ for $48 \mathrm{~h}$. The results of real-time PCR and western blotting analyses showed that miR-133b significantly inhibited the downregulated mRNA and protein expression of E-cadherin as well as the upregulated mRNA and protein expression of $\alpha$ SMA, fibronectin, Col3A1, and CTGF induced by TGF$\beta 1$ (Fig. 4A, B).

Immunofluorescence results showed that the fluorescence intensity of $\alpha$-SMA, Col3A1, fibronectin, and 

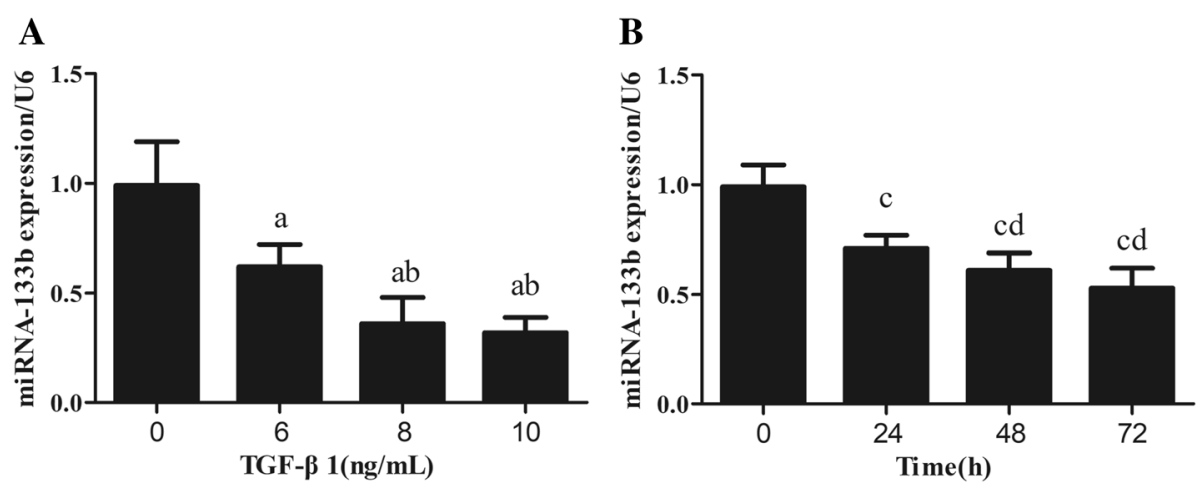

Fig. 1 Expression of miR-133b in HK2 cells stimulated with different concentrations of TGF- $\beta 1$ and at different time points. The experiment was performed in triplicate, and the expression of miR-133b was detected by real-time PCR. A The expression of miR-133b in HK2 cells stimulated with different concentrations of TGF- $\beta 1$. a: $P<0.05$ versus $0 \mathrm{ng} / \mathrm{mL}$ of TGF- $\beta 1$ group; $b: P<0.05$ versus $6 \mathrm{ng} / \mathrm{mL}$ of TGF- $\beta 1$ group. B The expression of miR-133b in HK2 cells stimulated with $8 \mathrm{ng} / \mathrm{mL}$ of TGF- $\beta 1$ at different time points. c: $P<0.05$ versus the $0-\mathrm{h}$ group; $\mathrm{d}$ : $P<0.05$ versus the 24 -h group
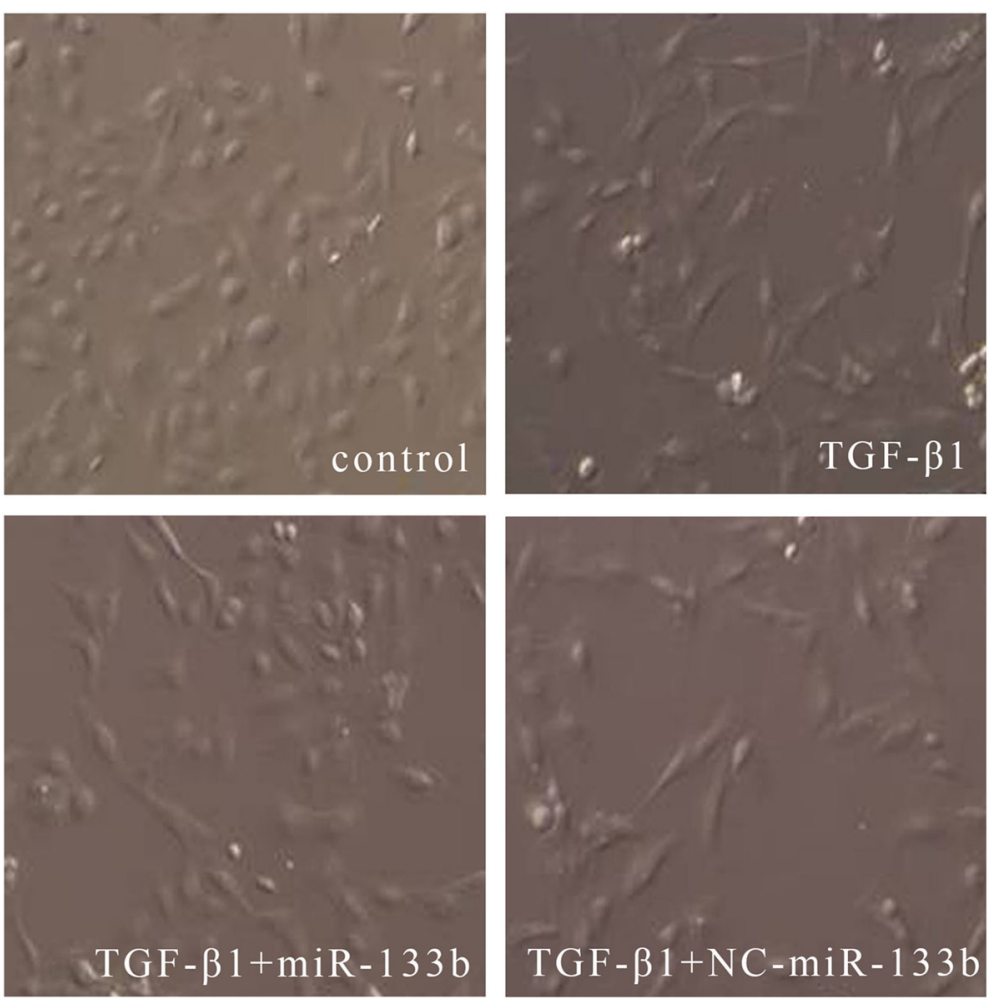

Fig. 2 Morphological changes in HK2 cells following different stimulation conditions. The morphological changes in cells were observed under an inverted microscope $(\times 100)$. Control: HK2 cells were cultured for $48 \mathrm{~h}$ without stimulation; TGF- $\beta 1$ : HK2 cells were stimulated with $8 \mathrm{ng} / \mathrm{mL}$ of TGF- $\beta 1$ for 48 h; TGF- $\beta 1+$ miR-133b: HK2 cells transfected with miR-133b were stimulated with $8 \mathrm{ng} / \mathrm{mL}$ of TGF- $\beta 1$ for 48 h; TGF- $\beta 1+N C-m i R:$ HK2 cells transfected with NC-miR were stimulated with $8 \mathrm{ng} / \mathrm{mL}$ of TGF- $\beta 1$ for $48 \mathrm{~h}$. HK2 cells exhibited a round or an oval shape. After stimulation with $8 \mathrm{ng} / \mathrm{mL}$ of TGF- $\beta 1$ for $48 \mathrm{~h}$, the cells became slender and fusiform, and their intercellular space significantly widened. While most cells transfected with miR-133b continued to maintain the morphology of epithelial cells, the NC-miR-transfected cells exhibited the morphology of fibroblasts 

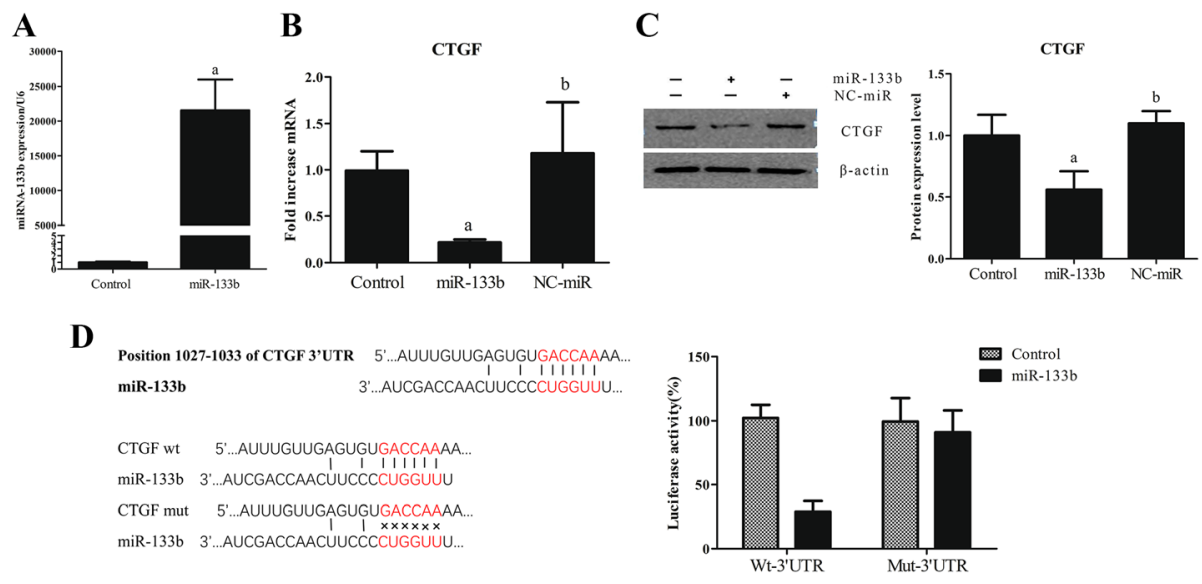

Fig. 3 CTGF is a direct target gene of miR-133b. Transient transfection of miR-133b mimic into HK2 cells for 48 h. A miR-133b expression was detected by real-time PCR. B CTGF expression was detected by real-time PCR. C CTGF expression was analyzed by western blotting. D Dualluciferase reporter assay confirmed CTGF as a direct target gene of miR-133b

CTGF increased and that of E-cadherin decreased in cells treated with TGF- $\beta 1$ as compared to that in control cells. Compared to cells stimulated with TGF- $\beta 1$ alone, the cells treated with TGF- $\beta 1$ and miR-133b showed a decrease in the fluorescence intensity of $\alpha$-SMA, Col3A1, fibronectin, and CTGF and an increase in the intensity of E-cadherin. Further, no significant change was observed in the fluorescence intensity of $\alpha$-SMA, Col3A1, E-cadherin, fibronectin, and CTGF between the cells treated with TGF- $\beta 1$ and NC-miR-133b and those treated with TGF- $\beta 1$ alone (Table 1 ; Fig. 4 C).

\section{Overexpression of CTGF reverses the protective effect of miR-133b}

The cells were transfected with CTGF for $48 \mathrm{~h}$, and then they were collected and analyzed by western blotting. Compared to HK2 cells transfected with GAPDH, cells transfected with $C T G F$ showed a significant upregulation in the expression of CTGF protein, thereby confirming the successful transfection of CTGF (Fig. 5A). We stimulated HK2 cells with $8 \mathrm{ng} / \mathrm{mL}$ of TGF- $\beta 1$ for $48 \mathrm{~h}$ and performed western blotting. We found that the miR133b overexpression group showed a significant downregulation in the expression of CTGF, $\alpha$-SMA, fibronectin, and Col3A1 and a significant upregulation in the expression of E-cadherin as compared to that in the control group. The cells overexpressing miR-133b and CTGF showed a significant increase in CTGF expression. CTGF could revert the inhibition of $\alpha$-SMA, fibronectin, and Col3A1 expression and the increase in Ecadherin expression mediated by miR-133b overexpression. Furthermore, the group overexpressing miR-133b and CTGF showed significantly higher CTGF and $\alpha$ SMA levels and significantly lower E-cadherin levels than the control group. The group overexpressing miR- 133b and GAPDH showed no significant difference in the expression of various proteins as compared to the miR-133b overexpression group (Fig. 5B).

The results of dual-luciferase reporter assay showed that the miR-133b mimic significantly decreased the luciferase activity of miRGLO-Wt-CTGF but had no significant effect on the luciferase activity of miRGLOMut-CTGF (Fig. 3D).

miR-133b inhibits renal interstitial fibrosis and reduces renal function loss in aged mice with UUO

The miR-133b or NC-miRNA transfection complex was administered to mice (UUO+miR-133b or UUO+NC$\mathrm{miR}$, respectively) by intravenous injection and the expression of miR-133b was detected by real-time PCR. At 7 and 14 days after UUO establishment, the expression of miR-133b was significantly higher in the UUO+miR$133 \mathrm{~b}$ group than that in the sham and $\mathrm{UUO}+\mathrm{NC}-\mathrm{miR}$ groups, thereby confirming the successful expression of miRNA-133b. The expression of miRNA-133b in the $\mathrm{UUO}+\mathrm{NC}-\mathrm{miR}$ group was significantly lower than that in the sham group (Fig. 6A).

PAS and Masson's trichrome staining techniques were performed to observe the morphology of the kidney tissue. Compared to the sham group, the UUO+NC-miR group showed interstitial edema, tubular dilatation, interstitial inflammatory cell infiltration, and a slight increase in collagen levels at 7 days after UUO surgery on the ureter ligation side of the kidney. At 14 days after UUO surgery, the interstitial cell population increased, and collagen expression was significantly upregulated. Compared to the UUO+NC-miR group, the UUO+miR133b group showed a significant decrease in the level of collagen in the renal interstitium at 7 and 14 days after UUO surgery (Fig. 6B). 

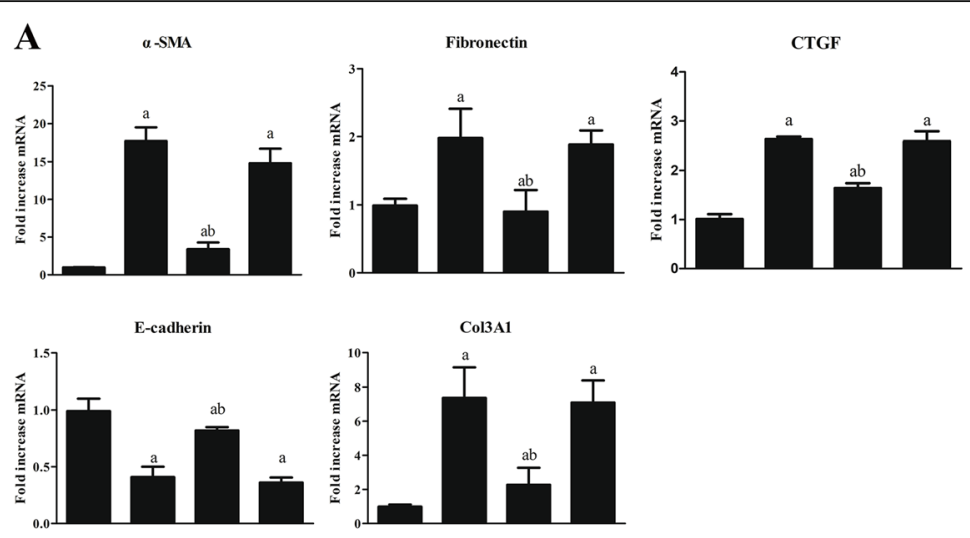

B
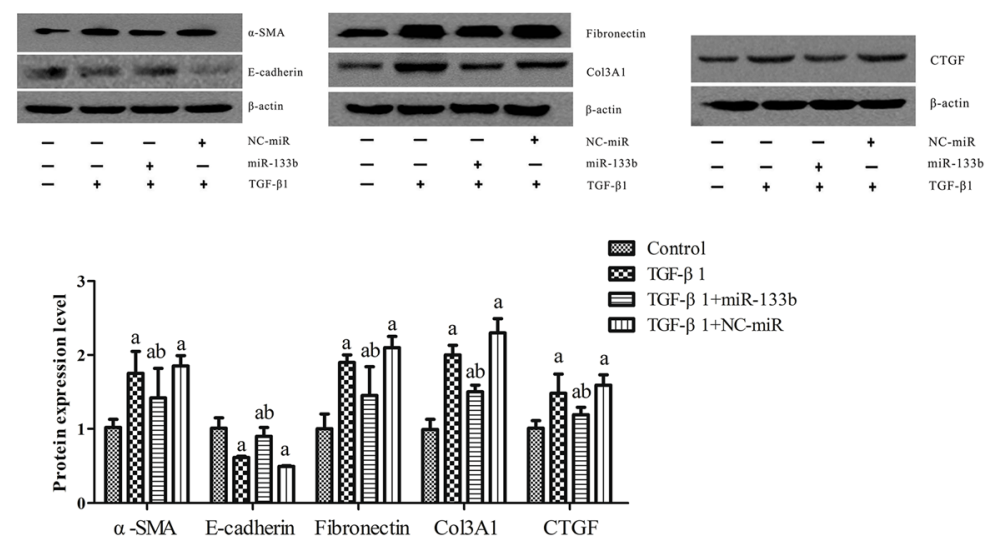

C

Control

TGF- $\beta 1$

TGF- $\beta 1+$ miR-133 TGF- $\beta 1+N C-m i R$
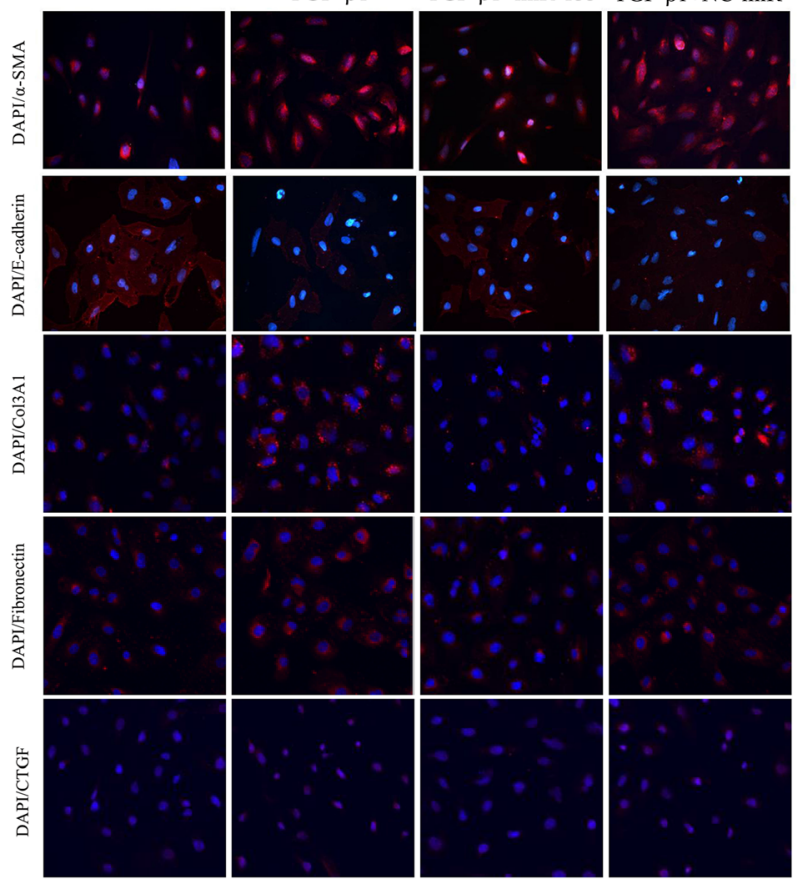

Fig. 4 miR-133b inhibited EMT of HK2 cells induced by TGF- 1 1. Normal (untransfected) HK2 cells or HK2 cells transfected with miR-133b mimic/ $\mathrm{NC}$-miR for $24 \mathrm{~h}$ were stimulated with $8 \mathrm{ng} / \mathrm{mL}$ of TGF- $\beta 1$ for $48 \mathrm{~h}$. A Real-time PCR results. B Western blotting results. C Cell immunofluorescence assay results. a: $P<0.05$ versus the control group; $b: P<0.05$ versus the TGF- $\beta 1$ group 
Table 1 Comparison of fluorescence intensity of a-SMA and E-cadherin in HK2 cells under different stimulation conditions

\begin{tabular}{|c|c|c|c|}
\hline \multirow[t]{2}{*}{ Group } & \multirow{2}{*}{$\begin{array}{l}\text { Number of } \\
\text { experiments }\end{array}$} & \multicolumn{2}{|c|}{ Average fluorescence intensity of cells } \\
\hline & & a-SMA & E-cadherin \\
\hline Control & 3 & $0.021 \pm 0.003$ & $0.175 \pm 0.028$ \\
\hline TGF- $\beta 1$ & 3 & $0.103 \pm 0.043^{*}$ & $0.013 \pm 0.001^{*}$ \\
\hline TGF- $\beta 1+m i R-133 b$ & 3 & $0.041 \pm 0.005^{*, \#}$ & $0.067 \pm 0.009^{* \text {,\# }}$ \\
\hline TGF- $\beta 1+N C-m i R$ & 3 & $0.092 \pm 0.004^{*}$ & $0.009 \pm 0.001^{*}$ \\
\hline
\end{tabular}

The immunofluorescence experiment was performed in triplicate, and five fields of vision were selected for each group. The fluorescence intensity of cells was observed under a confocal microscope $(\times 100) ;{ }^{*} P<0.05$ versus the control group; ${ }^{\sharp} P<0.05$ versus the TGF- $\beta 1$ group

We observed the relative area of collagen deposition in the kidney tissue and found it to be significantly higher in all animals with UUO than that in the sham group. The value observed for the UUO+miR-133b group was significantly lower than that obtained for the UUO+NC-miR group at 7 and 14 days after UUO surgery (Table 2).

The results of real-time PCR and western blotting analyses showed that the mRNA and protein levels of CTGF, fibronectin, Col3A1, and $\alpha$-SMA in the UUO+ $\mathrm{NC}$-miR group were significantly higher and that of Ecadherin were significantly lower than the values reported in the sham group. Further, the changes were more significant at 14 days after UUO surgery as compared to that observed 7 days after UUO surgery. At both 7 and 14 days after UUO surgery, the mRNA and protein levels of CTGF, fibronectin, Col3A1, and $\alpha$-SMA significantly decreased and that of E-cadherin significantly increased in the UUO+miR-133b group as compared to the values observed for the $\mathrm{UUO}+\mathrm{NC}-\mathrm{miR}$ group. However, the mRNA and protein levels of CTGF, fibronectin, Col3A1, and $\alpha$-SMA in the UUO+miR-133b group continued to be significantly higher and those of E-cadherin were significantly lower compared to the values for the sham group.

The results of the renal function test showed no significant difference in blood urea nitrogen (BUN) and serum creatinine $(\mathrm{sCr})$ levels between the three groups at day 0 . At day 7 following UUO surgery, BUN and $\mathrm{sCr}$ levels significantly increased in mice with UUO as compared to that in sham mice. The BUN level in the UUO+miR-133b group was significantly lower than that in the UUO+NC-miR group. At 14 days after UUO
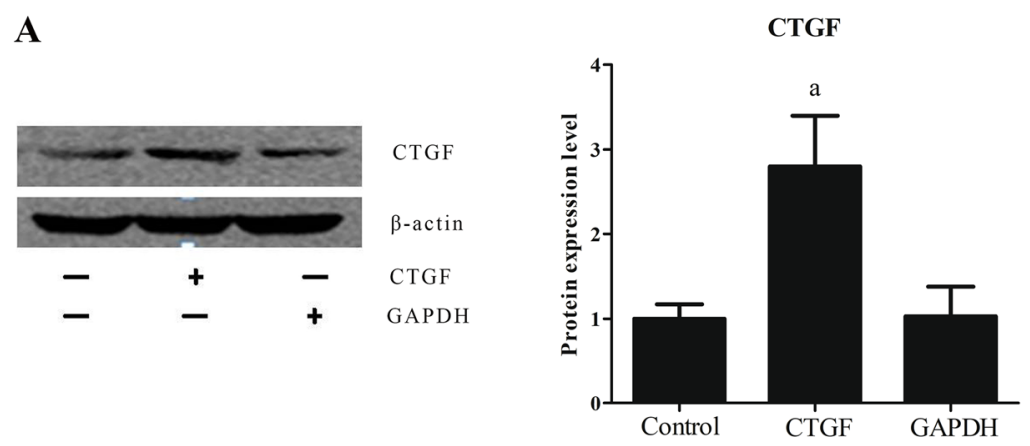

B
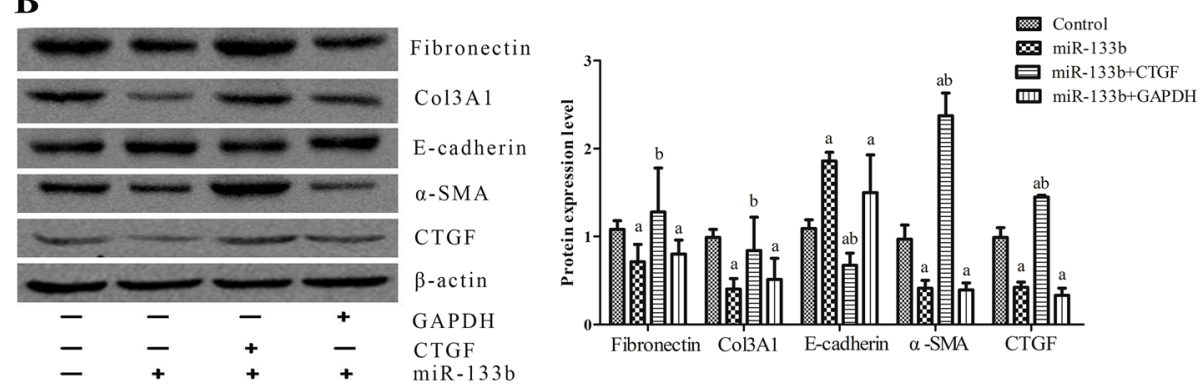

Fig. 5 miR-133b inhibited EMT of HK2 cells induced by TGF- $\beta 1$. A Western blotting results after transfection of HK2 cells with CTGF for 48 h. B Western blot analysis of HK2 cells stimulated with $8 \mathrm{ng} / \mathrm{mL}$ of TGF- $\beta 1$ for $48 \mathrm{~h}$. a: $P<0.05$ versus the control group; $b$ : $P<0.05$ versus the miR-133b group 

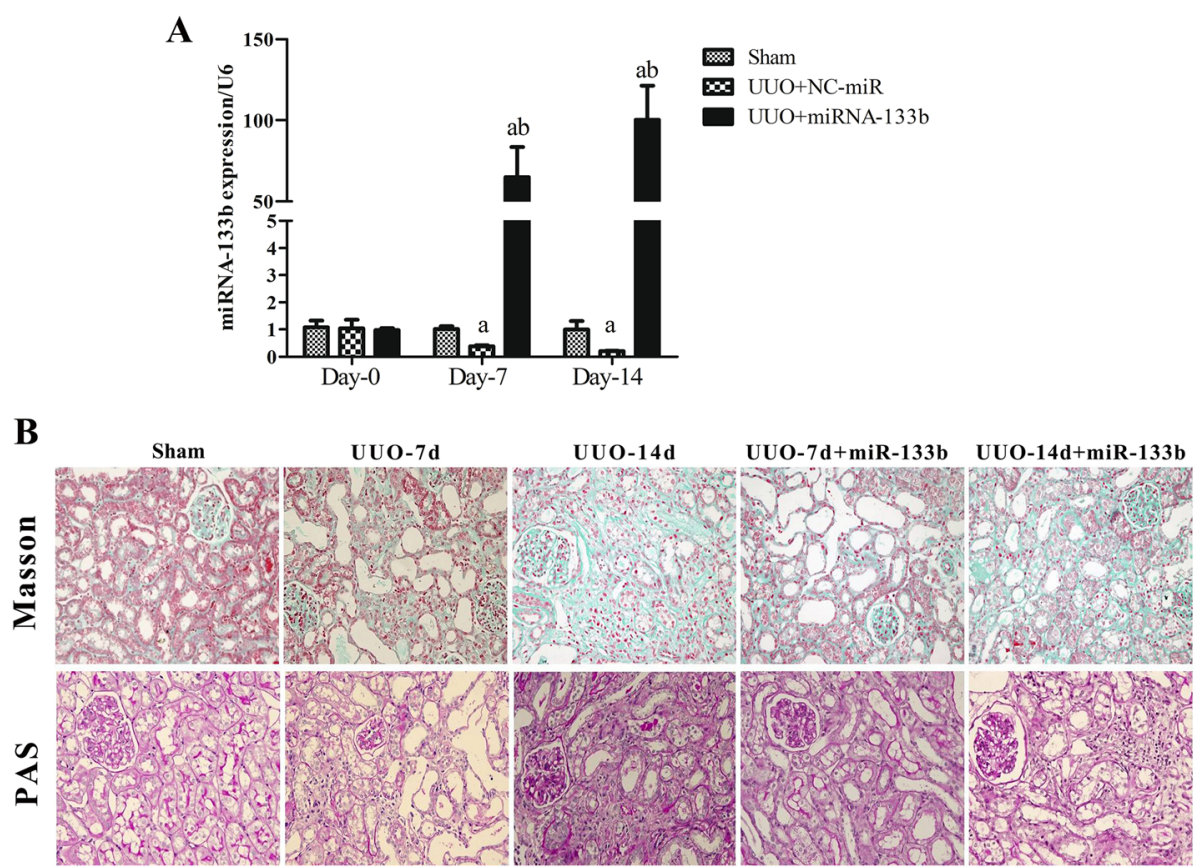

Fig. 6 miR-133b inhibited renal interstitial fibrosis in aged mice with UUO. Mice with UUO were administered with an intravenous injection of miR-133b or NC-miRNA transfection complex. A Real-time PCR analysis of miRNA-133b expression. B Masson's trichrome and PAS staining of renal tissues as observed under a light microscope $(\times 100)$. a: $P<0.05$ versus the sham group; $b$ : $P<0.05$ versus UUO+NC-miR group

surgery, BUN and sCr levels in UUO mice were significantly higher than those in the sham mice and the levels in the UUO+miR-133b group were significantly lower than the values reported for the UUO+NC-miR group (Table 3).

\section{Discussion}

Tubulointerstitial fibrosis is a chronic and progressive process which affects the kidney tissue during aging as well as in chronic kidney disease regardless of the underlying cause [12]. Kidney fibrosis is characterized by EMT

Table 2 Comparison of the relative area of collagen deposition in the kidney tissues of animals from each group

\begin{tabular}{lll}
\hline Group & Number & $\begin{array}{l}\text { Relative area of collagen deposition } \\
\text { (\%) }\end{array}$ \\
\hline Sham-7d & 4 & $4.10 \pm 0.3$ \\
Sham-14d & 4 & $4.09 \pm 0.3$ \\
UUO-7d+NC-miR & 5 & $38.17 \pm 5.67^{*}$ \\
UUO-14d+NC-miR & 5 & $82.52 \pm 7.10^{*}$ \\
UUO-7d+miR-133b & 5 & $17.83 \pm 4.16^{*, \#}$ \\
UUO-14d+miR- & 5 & $40.34 \pm 6.18^{*, \#}$ \\
133b & & \\
\hline
\end{tabular}

After Masson's trichrome staining, 10 fields sampled for each animal were observed under a light microscope $(\times 400)$, and the area of each field and the area of collagen fibers stained in green were measured by the Image-Pro Plus software. The relative area of collagen deposition was calculated as follows: area of collagen fibers stained in green/area of field of vision $\times 100 \%$. ${ }^{*} P<$ 0.01 versus the sham group; ${ }^{\#} P<0.01$ versus the UUO+NC-miR group of tubular epithelial cells [13] which contributes to both the destruction of the tubular epithelial compartment and the accumulation of interstitial fibroblasts [14]. TGF- $\beta$ signaling is thought to play a predominant role in this process [15]. CTGF is a direct downstream early response factor of TGF- $\beta$, also known to potentiate TGF- $\beta$ signaling by directly binding to TGF- $\beta 1$ through its CR domain [16].

Kidney aging is the basis for susceptibility and high incidence of kidney disease among the elderly. Renal interstitial fibrosis is an important pathological feature of kidney aging, and it is also the main pathogenic event which results in the progression of acute kidney injury to chronic kidney disease $[17,18]$. In our previous study, we found that miR-133b-3p was underexpressed in MSC-EVs of aged rats; however, miR-133b can inhibit the epithelialmesenchymal transition (EMT) of renal tubular cells induced by transforming growth factor (TGF)- $\beta 1$ [6]. We used a heterogeneous conjoined animal model and found that a younger blood environment could improve renal interstitial fibrosis in aging kidneys [19]. Therefore, we hypothesized that exosomes of bone marrow-derived mesenchymal stem cells might act as a humoral factor to affect renal interstitial fibrosis in the elderly. miRNA-133b can improve renal interstitial fibrosis in the elderly. The low expression of miRNA-133b in stem cell exosomes may be a cause of renal interstitial fibrosis in the elderly. Based on this hypothesis, we conducted this study. 
Table 3 Comparison of renal functions among different groups of animals

\begin{tabular}{|c|c|c|c|c|c|c|c|c|c|}
\hline \multirow[t]{2}{*}{ Groups } & \multicolumn{3}{|c|}{0 days } & \multicolumn{3}{|c|}{7 days } & \multicolumn{3}{|c|}{14 days } \\
\hline & $\bar{n}$ & BUN (mmol/L) & $\mathrm{sCr}(\mu \mathrm{mol} / \mathrm{L})$ & $\bar{n}$ & BUN (mmol/L) & $\mathrm{sCr}(\mu \mathrm{mol} / \mathrm{L})$ & $\bar{n}$ & BUN (mmol/L) & $\mathrm{sCr}(\mu \mathrm{mol} / \mathrm{L})$ \\
\hline Sham & 8 & $6.9 \pm 1.0$ & $17.4 \pm 0.7$ & 4 & $6.7 \pm 1.0$ & $17.2 \pm 0.7$ & 4 & $7.0 \pm 1.4$ & $17.3 \pm 1.0$ \\
\hline UUO+NC-miR & 10 & $6.9 \pm 1.0$ & $17.7 \pm 2.1$ & 5 & $13.8 \pm 1.7^{*}$ & $22.5 \pm 1.2^{*}$ & 5 & $18.3 \pm 1.5^{*}$ & $28.4 \pm 0.7^{*}$ \\
\hline UUO+miR-133b & 10 & $6.7 \pm 0.6$ & $17.1 \pm 1.9$ & 5 & $10.3 \pm 1.1^{*, \#}$ & $20.5 \pm 1.7^{*}$ & 5 & $14.1 \pm 1.3^{*, \#}$ & $23.2 \pm 1.5^{*, \#}$ \\
\hline
\end{tabular}

BUN blood urea nitrogen, $s C r$ serum creatinine; ${ }^{*} P<0.05$ versus the sham group; ${ }^{\#} P<0.05$ versus the UUO+NC-miR group

The miR-133b miRNA was first experimentally characterized in mice, and its homologs were identified in several other species, including invertebrates such as the fruit fly, Drosophila melanogaster [20]. In the human genome, miR-133 genes include miR-133a-1, miR-133a2 , and miR-133b located on chromosomes 18,20 , and 6 , respectively [21]. miR-133 is necessary for the proper development and function of skeletal and cardiac muscles, and its aberrant expression has been linked to many diseases associated with skeletal and cardiac muscles. It is identified as a key factor in cancer development [20-23] and is also known to alleviate cardiac fibrosis in many animal models [8-10]. Lentiviral transfection of miR133b was found to reduce renal interstitial fibrosis in aged mice with UUO [7] and renal fibrosis in diabetic rats [24]. In the present study, we induced high expression of miR-133b in the kidney tissue through intravenous injection of a miR-133b transfection complex. It was found that miR-133b downregulated the mRNA and protein expression of CTGF, fibronectin, Col3A1, and $\alpha$ SMA and upregulated the mRNA and protein levels of E-cadherin, thereby significantly alleviating renal interstitial fibrosis and reducing loss of renal function in aged mice with UUO. Thus, we reconfirmed the effect of miR-133b on geriatric renal interstitial fibrosis.

The effect of miR-133b on EMT induced by TGF- $\beta 1$ is controversial. miR-133b was previously shown to inhibit EMT of HK2 cells induced by TGF- $\beta 1[6,7]$. TGF- $\beta 1$ downregulated the expression of miR-133a/b in bladder smooth muscle epithelial cells in a concentrationdependent manner, and transfection with miR-133 mimics resulted in the attenuation of TGF- $\beta 1$-induced expression of $\alpha$-SMA, extracellular matrix subtype proteins, and growth factors associated with fibrosis [11]. Treatment of primary murine and human hepatic stellate cells with TGF- $\beta 1$ resulted in significant downregulation in the expression of miR-133a. On the other hand, overexpression of miR-133a in primary murine hepatic stellate cells was shown to decrease the expression of collagen [25]. However, miR-133b was found to be overexpressed in TGF$\beta 1$-treated HK2 cells, and miR-133b inhibition attenuated TGF- $\beta 1$-induced EMT of HK2 cells [24]. Our experiments confirmed the TGF- $\beta 1$-mediated downregulation of miR133 expression in a concentration- and time-dependent manner and showed that overexpression of miR-133b significantly inhibited the downregulation of mRNA and protein expression of E-cadherin as well as the upregulation of mRNA and protein levels of $\alpha$-SMA, fibronectin, Col3A1, and CTGF induced by TGF- $\beta 1$. The inhibitory effect of miR-133b on EMT of HK2 cells induced by TGF$\beta 1$ was further clarified.

Only few studies have evaluated the effect of target genes of miR-133b on the alleviation of tissue fibrosis. A single study on renal fibrosis confirmed Sirtuin-1 as a target of miR-133b in HK2 cells and showed that the inhibition of miR-133b expression resulted in the attenuation of TGF- $\beta 1$-induced EMT and renal fibrosis through the upregulation of Sirtuin-1 expression [24]. Further, CTGF was found to be a direct target of miR133 during EMT of cardiomyocytes [9] and bladder smooth muscle epithelial cells [11]. The overexpression of miR-133b significantly downregulated the mRNA and protein levels of CTGF, and CTGF overexpression could reverse the inhibitory effect of miR-133b on TGF- $\beta 1$ induced EMT of HK2 cells. Moreover, the results of the dual-luciferase reporter assay confirmed that CTGF was a direct target of miR-133b. Therefore, our study confirms for the first time that CTGF is a target of miR$133 \mathrm{~b}$ and is involved in amelioration of renal fibrosis.

CTGF not only potentiates TGF- $\beta$ signaling by directly binding to TGF- $\beta 1$, but also modifies various growth factors and cytokines. Each domain of CTGF can bind to multiple ligands, including insulin-like growth factor-1, fibronectin, bone morphogenetic factors, $\alpha 5 \beta 3$ integrin, low-density lipoprotein receptor-related protein 1, vascular endothelial growth factor (VEGF), Wnt, integrins, heparan sulfate proteoglycan, receptor-related proteins, and epidermal growth factor receptor [25]. These cytokines can participate in kidney aging and renal fibrosis through various signaling pathways. Therefore, miR-133b may play different biological roles by downregulating the expression of CTGF and by influencing the processes of kidney aging and renal fibrosis. These effects need to be confirmed through future studies.

\section{Conclusions}

We showed that CTGF was a target gene of miR-133b and was involved in ameliorating renal fibrosis. Further, we clarified that miR-133b-mediated inhibition of EMT of HK2 cells induced by TGF- $\beta 1$ resulted in the 
alleviation of renal interstitial fibrosis in aged mice with UUO. Improvement of renal interstitial fibrosis in the elderly can help delay kidney aging. These observations serve as a basic research evidence for the development of new drugs based on miR-133b for the amelioration of kidney aging and renal interstitial fibrosis.

\section{Supplementary Information}

The online version contains supplementary material available at https://doi. org/10.1186/s13287-021-02210-2.

\section{Additional file 1.}

\section{Abbreviations}

miRNA: MicroRNA; MSCs: Mesenchymal stem cells; EVs: Extracellular vesicles; MSC-EVs: MSC-derived extracellular vesicles; TGF- $\beta 1$ : Transforming growth factor- $\beta 1$; EMT: Epithelial-mesenchymal transition; HK2: Human renal proximal tubular epithelial; a-SMA: Alpha-smooth muscle actin; COL3A1: Alpha 1 chain of type III collagen; UUO: Unilateral ureteral obstruction; CTGF: Connective tissue growth factor; NC-miRNA: Negative control microRNA;

FITC: Fluorescein isothiocyanate; PAS staining: Periodic acid-Schiff staining; BUN: Blood urea nitrogen; sCr: Serum creatinine

\section{Acknowledgements}

Not applicable.

\section{Authors' contributions}

XS participated in the conceptualization, design, and coordination of this study. Animal experiments were conducted by DC, $Y Z, Q H$, and $Z Y$. Cell culture experiments were performed by DC, YW, and $Y Z$. Statistical analyses of the data were performed by DC, YW, and XS. The manuscript was written and revised by DC, YW, XS, GC, and MC. All authors read and approved the final manuscript.

\section{Funding}

This study was supported by a grant from the National Natural Science Foundation of China (81870463) and (92049103) and the Major State Basic Research Development Program of China (2013CB530800).

\section{Availability of data and materials}

The datasets used and/or analyzed during the current study will be available from the corresponding author upon reasonable request.

\section{Ethics approval and consent to participate}

Animal welfare was ensured, and all experimental procedures were carried out in accordance with the Guide for the Care and Use of Laboratory Animals (Ministry of Science and Technology of China, 2006).

\section{Consent for publication}

Not applicable.

\section{Competing interests}

The authors declare no competing interests.

Received: 9 August 2020 Accepted: 3 February 2021

Published online: 10 March 2021

\section{References}

1. Hommos MS, Glassock RJ, Rule AD. Structural and functional changes in human kidneys with healthy aging. J Am Soc Nephrol. 2017;28(10):2838-44.

2. Yang $\mathrm{HC}$, Rossini $\mathrm{M}, \mathrm{Ma} \mathrm{L}$, et al. Cells derived from young bone marrow alleviate renal aging. J Am Soc Nephrol. 2011;22(11):2028-36.

3. Matsui F, Babitz SK, Rhee A, et al. Mesenchymal stem cells protect against obstruction-induced renal fibrosis by decreasing STAT3 activation and STAT3-dependent MMP-9 production. Am J Physiol Renal Physiol. 2017; 312(1):F25-f32

4. Choi HY, Lee HG, Kim BS, et al. Mesenchymal stem cell-derived microparticles ameliorate peritubular capillary rarefaction via inhibition of endothelial-mesenchymal transition and decrease tubulointerstitial fibrosis in unilateral ureteral obstruction. Stem Cell Res Ther. 2015:6(1):18.

5. Rando TA. Stem cells, ageing and the quest for immortality. Nature. 2006; 441(7097):1080-6.

6. Wang Y, Fu B, Sun X, et al. Differentially expressed microRNAs in bone marrow mesenchymal stem cell-derived microvesicles in young and older rats and their effect on tumor growth factor- $\beta 1$-mediated epithelialmesenchymal transition in HK2 cells. Stem Cell Res Ther. 2015;6:185.

7. Wang Y, Guo YF, Fu GP, et al. Protective effect of miRNA-containing extracellular vesicles derived from mesenchymal stromal cells of old rats on renal function in chronic kidney disease. Stem Cell Res Ther. 2020;11(1):274.

8. Chen S, Puthanveetil P, Feng B, et al. Cardiac miR-133a overexpression prevents early cardiac fibrosis in diabetes. J Cell Mol Med. 2014;18(3):415-21.

9. Duisters RF, Tijsen AJ, Schroen B, et al. miR-133 and miR-30 regulate connective tissue growth factor: implications for a role of microRNAs in myocardial matrix remodeling. Circ Res. 2009;104(2):170-8.

10. Matkovich SJ, Wang W, Tu Y, et al. MicroRNA-133a protects against myocardial fibrosis and modulates electrical repolarization without affecting hypertrophy in pressure-overloaded adult hearts. Circ Res. 2010;106(1):166-75.

11. Duan LJ, Qi J, Kong XJ, et al. MiR-133 modulates TGF- $\beta 1$-induced bladder smooth muscle cell hypertrophic and fibrotic response: implication for a role of microRNA in bladder wall remodeling caused by bladder outlet obstruction. Cell Signal. 2015;27(2):215-27.

12. Humphreys BD. Mechanisms of renal fibrosis. Annu Rev Physiol. 2018;80 $309-26$.

13. Lovisa S, Lebleu VS, Tampe B, et al. Epithelial-to-mesenchymal transition induces cell cycle arrest and parenchymal damage in renal fibrosis. Nat Med. 2015;21(9):998-1009.

14. Zeisberg M, Kalluri R. Fibroblasts emerge via epithelial-mesenchymal transition in chronic kidney fibrosis. Front Biosci. 2008;13:6991-8.

15. Lamouille S, Xu J, Derynck R. Molecular mechanisms of epithelialmesenchymal transition. Nat Rev Mol Cell Biol. 2014:15(3):178-96.

16. Abreu JG, Ketpura NI, Reversade B, De Robertis EM. Connective-tissue growth factor (CTGF) modulates cell signalling by BMP and TGF-beta. Nat Cell Biol. 2002;4(8):599-604

17. Fiorentino M, Grandaliano G, Gesualdo L, Castellano G. Acute kidney injury to chronic kidney disease transition. Contrib Nephrol. 2018;193:45-54.

18. O'sullivan ED, Hughes J, Ferenbach DA. Renal aging: causes and consequences. J Am Soc Nephrol. 2017;28(2):407-20.

19. Huang $Q$, Ning $Y$, Liu D, et al. A young blood environment decreases aging of senile mice kidneys. J Gerontol A Biol Sci Med Sci. 2018;73(4):421-8.

20. Chen JF, Mandel EM, Thomson JM, et al. The role of microRNA-1 and microRNA-133 in skeletal muscle proliferation and differentiation. Nat Genet. 2006;38(2):228-33.

21. Li N, Zhou H, Tang Q. miR-133: a suppressor of cardiac remodeling? Front Pharmacol. 2018;9:903

22. Yu H, LU Y, Li Z, Wang Q. microRNA-133: expression, function and therapeutic potential in muscle diseases and cancer. Curr Drug Targets. 2014;15(9):817-28.

23. Liu Y, Liang Y, Zhang JF, Fu WM. MicroRNA-133 mediates cardiac diseases: mechanisms and clinical implications. Exp Cell Res. 2017;354(2):65-70.

24. Sun Z, Ma Y, Chen F, et al. miR-133b and miR-199b knockdown attenuate TGF- $\beta 1$-induced epithelial to mesenchymal transition and renal fibrosis by targeting SIRT1 in diabetic nephropathy. Eur J Pharmacol. 2018:837:96-104.

25. Toda N, Mukoyama M, Yanagita M, Yokoi H. CTGF in kidney fibrosis and glomerulonephritis. Inflamm Regen. 2018;38:14.

\section{Publisher's Note}

Springer Nature remains neutral with regard to jurisdictional claims in published maps and institutional affiliations. 